\title{
High-frequency 30-MHz sonography in preoperative assessment of tumor thickness of primary melanoma: usefulness in determination of surgical margin and indication for sentinel lymph node biopsy
}

\begin{abstract}
Background. High-frequency sonographic imaging has been used for the preoperative evaluation of primary malignant melanoma. In the present study, to identify the usefulness of 30-MHz sonography for determination of the surgical margin and indication for sentinel lymph node biopsy, the correlation between sonometric and histometric tumor thickness was investigated.

Methods. A total of 74 primary melanomas, in patients seen at the dermatology clinic, Shinshu University Hospital, from 1998 to 2006, were evaluated using high-frequency sonographic equipment with two probes $(15 \mathrm{MHz}$ and $30 \mathrm{MHz}$ ), and tumor thickness was measured using electronic calipers before surgical treatment. All the primary lesions were surgically excised and Breslow's tumor thickness was measured histologically.

Results. In 68 melanomas, excluding 2 lesions of melanoma in situ and 4 lesions with poor sonographic images, sonographic and histologic thickness showed good correlation $(r=0.887)$. Particularly, in 26 melanomas affecting the soles of the feet, sonographic and histologic thickness showed excellent correlation $(r=0.945)$. Regarding the T categorization, in which T1-T4 are divided at 1,2 , and $4 \mathrm{~mm}$ in thickness, the categories determined with sonometry corresponded very well to those determined with histometry. The correspondence was particularly excellent in thinner primary lesions with thickness around $1 \mathrm{~mm}$. We excised almost all these primary melanomas with surgical margins based on the sonometric thickness. In 22 patients with sonometric thickness more than $1 \mathrm{~mm}$, sentinel lymph node biopsy and/or radical lymphadenectomy was performed.

Conclusion. High-frequency sonography $(30-\mathrm{MHz})$ is very useful in the preoperative prediction of tumor thickness, particularly in thinner primary lesions, which allows us to

K. Hayashi $(\varangle) \cdot$ H. Koga $\cdot$ H. Uhara $\cdot$ T. Saida

Department of Dermatology, Shinshu University School of Medicine,

3-1-1 Asahi, Matsumoto 390-8621, Japan

Tel +81-263-37-2647, Fax +81-263-37-2646

e-mail: khayashi@shinshu-u.ac.jp
\end{abstract}

determine surgical margins and indication for sentinel lymph node biopsy.

Key words Malignant melanoma $\cdot$ High-frequency $30-\mathrm{MHz}$ sonography - Tumor thickness - Sentinel lymph node biopsy · Preoperative assessment

\section{Introduction}

The accurate preoperative evaluation of primary malignant melanoma is essential in determining appropriate treatment. Nowadays, several new methods for the evaluation of malignant melanoma have been introduced, such as highfrequency sonography, ${ }^{1}$ dermoscopy ${ }^{2}$ and sentinel lymph node biopsy, ${ }^{3}$ all of which can aid in less invasive treatment. A new staging system of malignant melanoma has been introduced, ${ }^{4}$ and sentinel lymph node biopsy is now recommended for patients with primary melanomas $1.0 \mathrm{~mm}$ or more in tumor thickness. Thus, in malignant melanoma, accurate preoperative evaluation of the tumor thickness is of great value in determining whether to carry out sentinel lymph node biopsy. Moreover, standard surgical margins of primary melanomas are now recommended based on the tumor thickness. Excisional biopsy is the best way to determine the precise tumor thickness, but this is an invasive surgical manipulation, which possibly damages the lymphatic drainage system. High-frequency sonography is a useful tool with which to evaluate the tumor thickness of primary melanoma preoperatively. The thickness is noninvasively evaluated by measuring the vertical distance between the lower edge of the entry echo and the deepest point of the posterior margin of the hypoechoic zone.

Until now, almost all sonographic studies have used 13or $20-\mathrm{MHz}$ sonography to evaluate the tumor thickness. Sonography with much higher frequency is better for the accurate evaluation of thinner primary melanomas because of its higher resolution. ${ }^{5}$ The purpose of this study was the evaluation of the value of high-frequency $30-\mathrm{MHz}$ sonography for the preoperative assessment of tumor thickness in 
Japanese patients with melanoma, in whom acral melanomas are the most prevalent.

\section{Patients and methods}

\section{Patients}

A total of 74 patients with malignant melanoma seen at the dermatology clinic, Shinshu University Hospital, from 1998 to 2006 were included in this study. The tumor thickness of the primary melanoma was evaluated using high-frequency sonographic equipment before surgical treatment.

\section{Ultrasonography equipment}

A high-frequency sonographic scanner (UX-02; Rion, Tokyo, Japan) with two probes (15-MHz and $30-\mathrm{MHz}$ ) was used to obtain B-scan images. These probes had mechanically activated $15-$ and $30-\mathrm{MHz}$ transducers housed in a built-in water chamber covered with a thin, disposable plastic membrane. The maximum dimensions of a typical two-dimension scan are 20 -mm width $\times 20$-mm depth for $15-\mathrm{MHz}$ and $10 \times 7.8 \mathrm{~mm}$ for $30-\mathrm{MHz}$. Standard sonographic gel was applied to the tumor surface. A serial sonographic examination was performed to visualize the echostructures of the entire lesion. In a few lesions showing hyperkeratosis, saline was applied onto the lesions for about $15 \mathrm{~min}$ before sonographic evaluation. This pretreatment substantially improved the image quality. The images were recorded with the gain curve enabling the best contrast between the tumor tissue and the surrounding tissue. Tumor thickness was measured using electronic calipers that allow a precision of $0.01 \mathrm{~mm}$. The maximal tumor thickness was evaluated by measuring the vertical distance between the lower edge of the entry echo and the deepest point of the posterior margin of the hypoechoic zone (Fig. 1). The 30-MHz probes were mainly used in this study because of their higher resolution in thinner lesions. However, $15-\mathrm{MHz}$ probes were also used for some lesions, particularly those thicker than $7 \mathrm{~mm}$.

\section{Histopathologic evaluation and statistical analysis}

All the primary lesions were surgically excised and routinely processed by fixation, dehydration, and embedding. Sections $4-\mu \mathrm{m}$-thick were cut from each tissue block and stained with hematoxylin and eosin. The tumor thickness was measured by the standard method using an ocular micrometer. The vertical distance from the uppermost level of the stratum granulosum in the epidermis to the lowest point of the tumor was recorded in millimeter units. ${ }^{6}$ The dermatopathologists who measured the tumor thickness did not know the thickness of the lesion estimated by sonography. The tumor thickness estimated by sonography was compared with the histologic tumor thickness, and the correlation was statistically evaluated with Pearson's correlation coefficient.

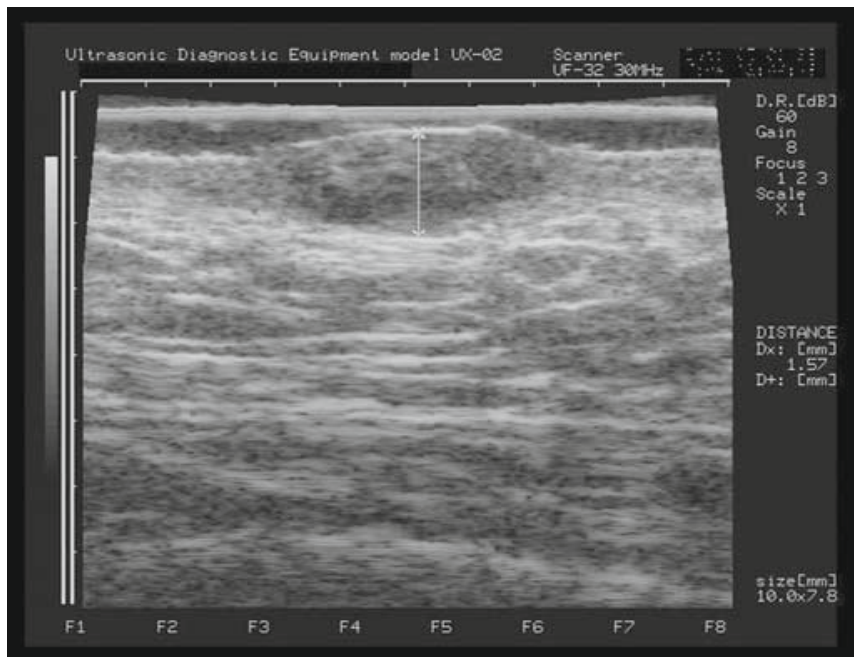

Fig. 1. The maximum tumor thickness was evaluated by measuring the vertical distance between the lower edge of the entry echo and the deepest point of the posterior margin of the hypoechoic zone

\section{Results}

A total of 74 primary melanomas including melanoma in situ were examined with the high-frequency sonographic equipment. In 70 lesions sonography showed good to excellent hypoechoic structures, but in the remaining 4 lesions, the sonographic images were poor for evaluating the thickness. Poor images were obtained in 2 melanomas on the soles of the feet, 1 melanoma on the nose, and 1 on the finger. In 1 melanoma on the foot, the poor image was probably due to a very thick cornified layer. In the remaining 3 lesions, however, we cannot give a clear explanation for the poor images. Two of the 70 melanomas showing hypoechoic structures were histopathologically diagnosed as melanoma in situ. In 1 melanoma on the ear, overestimation of the thickness was caused by dense lymphocytic infiltration in the dermis, suggesting a regression phenomenon. Apart from this case, the difference between the sonometric thickness and histometric thickness was more than $2 \mathrm{~mm}$ in only 5 melanomas; sonographic overestimation occurred in 3 melanomas affecting the head, nail apparatus, and genitalia, and sonographic underestimation was seen in 2 melanomas affecting the nail apparatus and an interdigital area.

In 68 of the 70 sonographically well-visualized melanomas, excluding 2 lesions of melanoma in situ, sonographic and histologic thickness showed good correlation $(r=0.887$; $P<0.0001$; Fig. 2). With regard to 26 melanomas affecting the sole of the foot, the sonographic and histologic thickness showed much better correlation $(r=0.945 ; P<0.0001$; Fig. 3). The 68 sonographically well-visualized melanomas were sonographically and histologically classified into the $\mathrm{T}$ categories proposed by the International Union against Cancer (UICC), in which the dividing points are 1,2, and $4 \mathrm{~mm}$ in thickness. Results of the categorization of all these melanomas, which revealed excellent correlation between the sonographic and histologic thickness, are shown in 
Table 1 . The predictive value was particularly good in 15 lesions with sonometric thickness of $1 \mathrm{~mm}$ or less; 13 lesions were correctly categorized by sonography and only 2 lesions were categorized as having a thickness of 1.01 to $2 \mathrm{~mm}$. Note that 2 lesions, 1 lesion on the foot with a histometric thickness of $1.3 \mathrm{~mm}$ and 1 of the nail apparatus with a histometric thickness of $2.5 \mathrm{~mm}$, were categorized as having a thickness of $1 \mathrm{~mm}$ or less by sonography. Concerning melanomas affecting only the sole of the foot, the correlation between the sonometric and histologic $\mathrm{T}$ categorizations was really excellent (Table 2). All the 5 lesions with a histologic thickness of $1 \mathrm{~mm}$ or less were correctly categorized

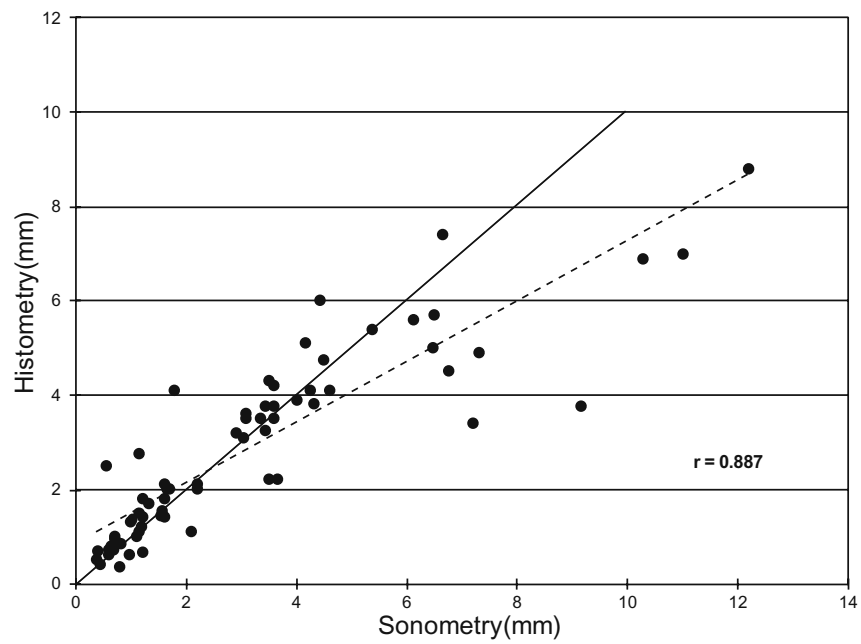

Fig. 2. Graph showing the scatter plot of sonometric and histometric measurements of tumor thickness in 68 primary melanomas $(r=0.887$; $P<0.0001)$. Dotted line, linear regression line; solid line, sonometry $=$ histometry by sonography. Each of 8 melanomas with a histologic thickness of 1.01 to $2 \mathrm{~mm}$, each of 6 melanomas with thickness of 2.01 to $4 \mathrm{~mm}$, and each of 7 melanomas with thickness of more than $4 \mathrm{~mm}$ were underestimated by one rank in the $\mathrm{T}$ categorization by sonography.

Almost all the primary melanomas in the patients enrolled in this study were excised with surgical margins based on the thickness predicted by sonography, and no

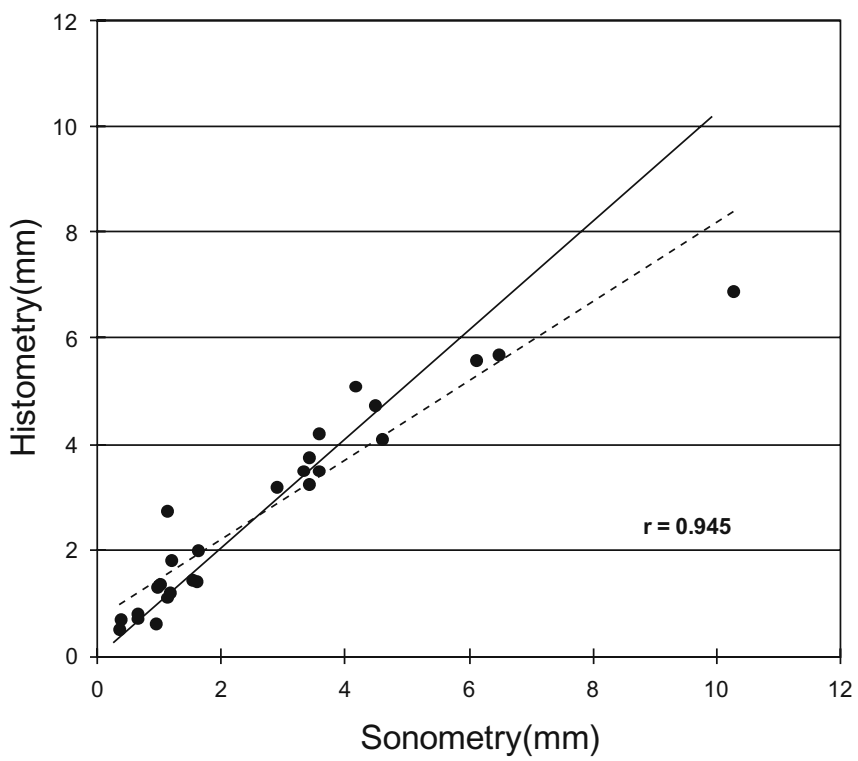

Fig. 3. Graph showing the scatter plot of sonometric and histometric measurements of tumor thickness in 26 melanomas on the sole of the foot $(r=0.945 ; P<0.0001)$. Dotted line, linear regression line; solid line, sonometry $=$ histometry

Table 1. Concordance of the $\mathrm{T}$ classification categories between sonometric and histometric thickness in 68 melanomas

\begin{tabular}{|c|c|c|c|c|c|c|}
\hline \multirow{2}{*}{$\begin{array}{l}\text { Sonometric } \\
\text { classes }\end{array}$} & \multicolumn{5}{|c|}{ Histometric classes } & \multirow{2}{*}{$\begin{array}{l}\text { Predictive value } \\
(\%)\end{array}$} \\
\hline & $\leq 1 \mathrm{~mm}$ & $1.01-2 \mathrm{~mm}$ & $2.01-4 \mathrm{~mm}$ & $>4 \mathrm{~mm}$ & Total & \\
\hline$\leq 1 \mathrm{~mm}$ & 13 & 1 & 1 & 0 & 15 & 86.7 \\
\hline $1.01-2 \mathrm{~mm}$ & 2 & 13 & 2 & 1 & 18 & 72.2 \\
\hline $2.01-4 \mathrm{~mm}$ & 0 & 2 & 12 & 2 & 16 & 75 \\
\hline$>4 \mathrm{~mm}$ & 0 & 0 & 4 & 15 & 19 & 78.9 \\
\hline Total & 15 & 16 & 19 & 18 & 68 & \\
\hline
\end{tabular}

Table 2. Concordance of the $\mathrm{T}$ classification categories between sonometric and histometric thickness in 26 melanomas on the sole of the foot

\begin{tabular}{lllllll}
\hline \multicolumn{2}{l}{\begin{tabular}{l} 
Predictive values of sonometry \\
\hline $\begin{array}{l}\text { Sonometric } \\
\text { classes }\end{array}$
\end{tabular}} & \multicolumn{2}{l}{ Histometric classes } & & \multicolumn{2}{l}{$\begin{array}{l}\text { Predictive value } \\
(\%)\end{array}$} \\
\cline { 2 - 6 } & $\leq 1 \mathrm{~mm}$ & $1.01-2 \mathrm{~mm}$ & $2.01-4 \mathrm{~mm}$ & $>4 \mathrm{~mm}$ & Total & \\
\hline$\leq 1 \mathrm{~mm}$ & 5 & 1 & 0 & 0 & 6 & 83.3 \\
$1.01-2 \mathrm{~mm}$ & 0 & 7 & 1 & 0 & 8 & 87.5 \\
$2.01-4 \mathrm{~mm}$ & 0 & 0 & 5 & 1 & 6 & 83.3 \\
$>4 \mathrm{~mm}$ & 0 & 0 & 0 & 6 & 6 & 100 \\
Total & 5 & 8 & 6 & 7 & 26 & \\
\hline
\end{tabular}


additional resection was needed in any cases. Twenty-two patients with melanomas with sonometric thickness of more than $1 \mathrm{~mm}$ and 1 patient with melanoma of the nail apparatus of the finger with sonometric thickness less than $1 \mathrm{~mm}$ but clinically estimated to be thicker than $1 \mathrm{~mm}$ underwent sentinel lymph node biopsy and/or radical lymphadenectomy. In the first 3 cases, we used only blue dye to identify the sentinel node, immediately followed by radical lymphadenectomy, which revealed micrometastasis in one case. In the following 2 patients, we used scintigram as well as blue dye to identify the sentinel node, also immediately followed by radical lymphadenectomy, which revealed no micrometastasis. In the remaining 18 patients, we used blue dye, scintigram, and a gamma probe to identify the sentinel node. In 7 of these 18 patients, micrometastases were found in the sentinel node(s) and radical lymphadenectomy was performed. Except for 1 case, all the cases with positive sentinel node(s) were more than $1 \mathrm{~mm}$ in sonometric thickness. However, in 1 melanoma affecting the nail apparatus, the quality of sonography was poor, in which the sonometric thickness was shown as less than $1 \mathrm{~mm}$, but the histologic thickness was $2.5 \mathrm{~mm}$, and radical lymphadenectomy was performed. In this patient, micrometastasis was detected in the sentinel node.

\section{Discussion}

In malignant melanoma, determination of the tumor thickness of the primary lesions and knowledge of the histopathologic status of the sentinel lymph node(s) are essential in determining further management and predicting the prognosis of the patient. ${ }^{7}$ In the present staging system of melanoma proposed by the UICC, tumor thickness of $1.00 \mathrm{~mm}$ is the dividing point between the T1 and T2 classifications. Moreover, $1.00-\mathrm{mm}$ thickness is the critical point of the indication for sentinel lymph node biopsy. ${ }^{4}$ Tumor thickness is formally determined by Breslow's method, ${ }^{6}$ using tissue sections after excision of the primary lesion. In general, the first step in the treatment of melanoma is to perform excisional biopsy of the primary lesion, by which tumor thickness is determined. A safe surgical margin is decided mainly based on the thickness. Furthermore, sentinel lymph node biopsy is adopted when the thickness is $1 \mathrm{~mm}$ or more or ulceration is present. However, excisional biopsy is invasive and possibly damages the local lymphatic drainage system. Recently, we experienced a patient with melanoma on the cheek who underwent a sentinel lymph node biopsy. In this patient, first, radiotracer was injected around the primary lesion and three lymph nodes were identified with scintigraphy; however, a second scintigraphy performed 1 week later identified only one lymph node, even though the same amount of radiotracer was injected to the same place around the primary melanoma. This experience indicates that minimal surgical manipulation, such as a local injection, could change the lymphatic drainage system. Excisional biopsy may have a much greater effect on the lymphatic drainage. In addition, excisional biopsy is difficult when the primary lesion is very wide or when it affects some anatomically critical sites such as the sole of the foot. Therefore, it would be of great value in the management of melanoma if the tumor thickness could be assessed preoperatively by any noninvasive methods.

As a noninvasive method, high-frequency sonography has been used for in vivo measurements of melanoma thickness. Good correlations between the sonometric and histopathologic thickness have been reported. ${ }^{1,8-12}$ Most of these sonographic studies used $13-$ or $20-\mathrm{MHz}$ probes. Salmhofer et al. $^{13}$ found that loss of skin tension after excision led to an increase in the measured tumor thickness because of spherical retraction of the specimen, but subsequent fixation, dehydration, and embedding reversed this effect, so that altogether, histopathologically assessed tumor thickness was only slightly lower than ultrasound-derived in vivo thickness before excision. They concluded that these findings led to a good overall correlation between sonographic and histopathologic measurements of tumor thickness. Weichenthal et al. ${ }^{14}$ reported that tumor thickness values overestimated by sonography may also reflect the more precise detection of the thickest part within the tumor by real-time thorough sonographic imaging of the whole lesion.

In contrast, several reports concluded that there were statistically significant differences between sonometry with $20-\mathrm{MHz}$ probes and histometry. ${ }^{15,16}$ Moreover, Semple et al. ${ }^{17}$ reported that sonography using much higher frequency, 40 to $60 \mathrm{MHz}$, would not replace the need to perform a biopsy of primary melanomas for the evaluation of the tumor thickness. But in their report only ten melanomas were evaluated and they mentioned that the article was not a formal assessment of diagnostic test validity. ${ }^{17}$

More recently, Guitera et al. ${ }^{5}$ reported that the measurement of thickness by $75-\mathrm{MHz}$ ultrasonography was highly reliable in invasive melanomas; the correlation between the sonographic and histologic thickness was very high $(r=$ 0.908).

In the present study, we used 30-MHz sonography and found a good correlation between the sonographic and histologic thickness $(r=0.887)$ in all melanomas; the correlation was much higher $(r=0.945)$ in melanomas affecting the sole of the foot, which is the most prevalent site of melanoma in non-Caucasians, including Japanese. The T categorization was also well correlated between sonographic and histopathologic evaluation. Pellacani and Seidenari ${ }^{10}$ combined 20-MHz sonography and videomicroscopy and proposed an algorithm for the in vivo distinction of thick and thin melanomas, which was useful to avoid the overestimation of tumor thickness, especially in lesions with a thickness of more than $1 \mathrm{~mm}$.

The data of the present study using 30-MHz sonography revealed that, in most lesions, the sonometric thickness consistently predicted the authentic histometric thickness; sonometric and histometric classifications were the same in $86.7 \%$ of the lesions with a thickness of $1 \mathrm{~mm}$ or less and in $72.2 \%$ of the lesions with a thickness of $1.01-2 \mathrm{~mm}$. The concordance rate was much higher in melanomas affecting the sole of the foot; the rate was $83.3 \%$ for lesions with a 
thickness of $1 \mathrm{~mm}$ or less and $87.5 \%$ for lesions with a thickness of 1.01-2 mm. In the present study, not all patients with a primary lesion of more than $1 \mathrm{~mm}$ in sonometric thickness underwent sentinel lymph node biopsy and/or radical lymphadenectomy, either because of the presence of distant metastases or because of refusal of further treatment by the patients. Twenty-two patients with primary lesions of sonometric thickness more than $1 \mathrm{~mm}$ underwent sentinel lymph node biopsy and/or radical lymphadenectomy. All primary melanomas in these 22 patients were also more than $1 \mathrm{~mm}$ in thickness by histometry.

In our present study, tumor thickness was reliably predicted in thinner melanomas of around $1 \mathrm{~mm}$. Particularly in melanomas on the sole of the foot, a much higher correlation $(r=0.945)$ between sonographic and histometric thickness was obtained. Thus, sonography is certainly very helpful in the preoperative estimation of tumor thickness in non-Caucasian patients with melanoma.

Semple et al. ${ }^{17}$ described that thick cornified layers can act as a virtual block to the ultrasound beam and thus, they excluded melanomas on the sole from their study. In the present study, we applied saline to the hyperkeratotic lesions in order to macerate the cornified layers, except for the first patient with a lesion on the sole. In this first patient, we could not obtain a clear image. In subsequent patients we applied saline to macerate the cornified layer. With this pretreatment, hyperkeratotic melanomas on the sole gave good echostructures, permitting us to accurately evaluate sonographic thickness. The $30-\mathrm{MHz}$ sonography has great impact on the preoperative planning of melanoma management because, in most cases, tumor thickness can be precisely predicted, especially in thinner lesions around $1 \mathrm{~mm}$ in thickness, which is the critical point for the indication of sentinel lymph node biopsy.

We experienced one melanoma on the nail apparatus of a finger that was sonographically $0.55 \mathrm{~mm}$ in thickness; however, from its clinical features, we estimated that the histological thickness would be more than $1 \mathrm{~mm}$. In this patient, we performed amputation of the finger and radical lymphadenectomy. The histological thickness was $2.5 \mathrm{~mm}$ and a positive sentinel node was detected in the subclavian area. Although such a case is rare, we must respect the clinical information if there are any conflicts between the sonographic and clinical data.

Finally, we propose the following strategy for the management of malignant melanoma. The first step is precise clinical examination. The second step is dermoscopic evaluation, which is essential in diagnosing melanoma, particularly early melanoma affecting the acral volar skin. ${ }^{18}$ The third step is the sonographic evaluation of the tumor thickness by using $30-\mathrm{MHz}$ sonography, which allows us to determine an adequate surgical margin for the primary melanoma, along with the indication for sentinel lymph node biopsy. This strategy is highly recommended because it is essentially noninvasive.

\section{Conflict of interest statement}

No author has any conflict of interest.

Acknowledgments This study was supported in part by a Grant-in-Aid for Cancer Research $(15-10,19-7)$ from the Ministry of Health, Labor, and Welfare of Japan.

\section{References}

1. Hoffmann K, Jung J, el Gammal S, et al. (1992) Malignant melanoma in 20-MHz B scan sonography. Dermatology 185:49-55

2. Argenziano G, Soyer HP, Chimenti S, et al. (2003) Dermoscopy of pigmented skin lesions: results of a consensus meeting via the Internet. J Am Acad Dermatol 48:679-693

3. Morton DL, Wen DR, Wong JH, et al. (1992) Technical details of intraoperative lymphatic mapping for early stage melanoma. Arch Surg 127:392-399

4. Balch CM, Buzaid AC, Soong SJ, et al. (2001) Final version of the American Joint Committee On Cancer Staging system for cutaneous melanoma. J Clin Oncol 19:3635-3648

5. Guitera P, Li L, Crotty K, et al.(2008) Melanoma histological Breslow thickness predicted by $75-\mathrm{MHz}$ ultrasonography. $\mathrm{Br} \mathrm{J}$ Dermatol 159:364-369

6. Breslow A (1970) Thickness, cross-sectional areas and depth of invasion in the prognosis of cutaneous melanoma. Ann Surg 172:902-908

7. Faries MB, Morton DL (2007) Surgery and sentinel lymph node biopsy. Semin Oncol 34:498-508

8. Lassau N, Spatz A, Avril MF, et al. (1997)Value of high-frequency US for preoperative assessment of skin tumors. Radiographics 17:1559-1565

9. Serrone L, Solvetti FM, Thorel MF, et al. (2002) High frequency ultrasound in the preoperative staging of primary melanoma: a statistical analysis. Melanoma Res 12:287-290

10. Pellacani G, Seidenari S (2003) Preoperative melanoma thickness determination by $20-\mathrm{MHz}$ sonography and digital videomicroscopy in combination. Arch Dermatol 139:293-298

11. Bessoud B, Lassau N, Koscielny S, et al. (2003) High-frequency sonography and color doppler in the management of pigmented skin lesions. Ultrasound Med Biol 29:875-879

12. Lassau N, Lamuraglia M, Koscielny S, et al. (2006) Prognostic value of angiogenesis evaluated with high-frequency and colour doppler sonography for preoperative assessment of primary cutaneous melanomas: correlation with recurrence after 5 year followup period. Cancer Imaging 6:24-29

13. Salmhofer W, Rieger E, Soyer HP, et al. (1996) Influence of skin tension and formalin fixation on sonographic measurement of tumor thickness. J Am Acad Dermatol 34:34-39

14. Weichenthal M, Mohr P, Breitbart EW (2001) The velocity of ultrasound in human primary melanoma tissue-implications for the clinical use of high resolution sonography. BMC Dermatol 1:1 Epub 2001 Jun 28

15. Tacke J, Haagen G, Hornstein OP, et al. (1995) Clinical relevance of sonometry-derived tumor thickness in malignant melanoma - a statistical analysis. Br J Dermatol 132:209-214

16. Partsch B, Binder M, Puspok-Schwartz M, et al. (1996) Limitations of high frequency ultrasound in determining the invasiveness of cutaneous malignant melanoma. Melanoma Res 6:395-398

17. Semple JL, Gupta AK, From L, et al. (1995) Does high frequency (40-60 MHz) ultrasound imaging play a role in the clinical management of cutaneous melanoma? Ann Plast Surg 34:599-606

18. Saida T, Miyazaki A, Oguchi S, et al. (2004) Significance of dermoscopic patterns in detecting malignant melanoma on acral volar skin: Results of a multi-center study in Japan. Arch Dermatol 140:1233-1238 